\title{
Beziehungen statt Produkte
}

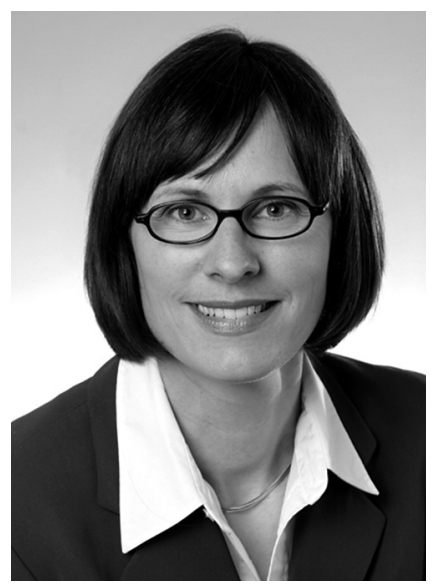

VON BRIGITTE REISER

Dr. Brigitte Reiser ist DiplomVerwaltungswissenschaftlerin und als Beraterin für Non-ProfitOrganisationen tätig. Sie ist spezialisiert auf die Themen Stakeholder-Management und Social Media für gemeinnützige Träger im Sozialsektor. Über beide Themen informiert sie regelmäßig in ihrem Fach-Weblog (http:// blog.nonprofits-vernetzt.de) und in Vorträgen und Publikationen. www.stakeholder-management.de

\author{
Die demografische Entwicklung, die Sparbemühungen \\ der öffentlichen Haushalte und die Wünsche der Nutzer \\ werden soziale Organisationen und Unternehmen \\ zwingen, stärker die Bürgerinnen und Bürger bereits \\ bei der Planung von sozialen Hilfen einzubeziehen. \\ Ein Hindernis bei dieser Koproduktion stellen vielfach \\ noch die Leistungsvereinbarungen mit der öffentlichen \\ Hand und den Kostenträgern dar, die ohne Bürgerinnen \\ und Bürger ausgehandelt und umgesetzt werden.
}

Koproduktion in den sozialen Diensten bedeutet, dass Bürger an der Planung und Erstellung von Dienstleistungen mitwirken. An der Produktion sozialer Dienste sind Bürgerinnen und Bürger als Nutzer, Angehörige und Freiwillige schon heute beteiligt. Allerdings fehlt ihnen in der Regel die Möglichkeit, die angebotenen Dienstleistungen inhaltlich mitzugestalten. Auch wenn Bürger durch ein Persönliches Budget ermächtigt werden und sich der einzelne sein Dienstleistungsangebot selbst zusammenstellen kann - zumindest in der Theorie -, so bleibt er dabei doch Konsument vorgefertigter Leistungen: Er hat zumeist keinen Einfluss auf das "Service Design« des jeweiligen Diensteangebots.

Koproduktion und die Beteiligung von Bürgern im Sozialbereich muss sich auf den ganzen Zyklus eines Leistungsangebots, also auch auf die Planungsund Ausgestaltungsphase beziehen. Nur so erhält der einzelne die Möglichkeit, über das »Wie« in der Pflege, Betreuung und Behandlung mitzubestimmen.

Umgekehrt profitieren soziale Dienste von einer Einbeziehung der Bürger in Planungsprozesse. Die Qualität sozialer Dienstleistungen offenbart sich gerade in der Nutzungsphase - so dass ohne eine Beteiligung bestehender und zukünftiger Nutzer keine effektive Dienstleistung konzipiert werden kann. Es fehlen in die- sem Fall das Nutzungswissen und die Nutzungserfahrungen der betroffenen Bürger. Statt auf fertige Dienstleistungsprodukte zu setzen, sollten Austauschprozesse mit den Stakeholdern in den Mittelpunkt der Aufmerksamkeit sozialer Dienste treten.

Im Rahmen solcher Austauschprozesse sollten die Leistungen einer Einrichtung von Mitarbeitern und Bürgern - Nutzern, Freiwilligen, Angehörigen, Quartiersbewohnern gemeinsam geplant und gestaltet werden. Bürger wachsen beim sogenannten »Co-Design « über die Rolle des passiven Subjektes hinaus, dessen Prioritäten durch Befragungen und Konsultationsprozesse eruiert werden. Sie werden nun zu gleichwertigen Partnern im Gestaltungsprozess (Sanders/Stappers 2008).

Wichtig ist beim Co-Design die Offenheit der Fragestellung zu Beginn des Prozesses: Es geht hier nicht darum, vorher festgelegte Produkte und Dienstleistungen auszugestalten, sondern zu fragen, auf welche Weise ein vorliegendes Problem gelöst werden kann. Aus dieser Fragestellung heraus werden Ideen, Konzepte und Prototypen gemeinschaftlich und iterativ - also immer wieder hinterfragend und verbessernd - entwickelt.

Die Prototypen spielen dabei eine wichtige Rolle, weil sie es den involvierten Stakeholdern erlauben, die 
entwickelten Lösungen zu nutzen und auszutesten. Im Idealfall werden die Prototypen dort erprobt, wo die (potenziellen) Nutzer anzutreffen sind, deren Feedback und Verbesserungsvorschläge an Ort und Stelle in die Weiterentwicklung des Prototypen einfließen (»Prototyping in the Wild «) (Hagen/Robertson 2012, 79).

Eine Studie der britischen »new economics foundation (nef) « vom Dezember 2012 analysiert, welche Aspekte die Beteiligung von Bürgern fördern (nef 2012). Auf der Ebene der Organisation muss die Führung gesammelt hinter dem Ziel der Koproduktion stehen und den Mitarbeitern signalisieren, dass Koproduktion zum »default way of working ", also zum Arbeitsstandard in der Einrichtung, werden soll (nef 2012, 2). Zu diesem Zweck braucht die Führung den regelmäßigen Austausch mit den Nutzern und Unterstützern der Einrichtung. Statt einer Steuerung auf »Armeslänge « ist ein enger Kontakt mit den betroffenen Bürgerinnen und Bürgern angesagt.

An der Organisationsbasis muss die Beteiligung von Bürgern alltäglich und in die Strukturen und Prozesse der Einrichtung integriert werden (Reiser 2012). Mitarbeiter brauchen dafür entsprechende Schulungen. Auch die Stellenbeschreibungen sollten jene Kompetenzen und Aufgaben umfassen, die für koproduktives Arbeiten notwendig sind (nef 2012, 5). Insbesondere der Fokus auf »Beziehungen «statt auf »Produkte«, auf gleichgewichtige Machtverhältnisse gegenüber Bürgern und auf die Notwendigkeit, dass Mitarbeiter selbst im Stadtteil gut vernetzt sind, sollte sich hier wiederfinden. Nur gut vernetzte Einrichtungen und Mitarbeiter können ihren Zielgruppen beim Netzwerkaufbau helfen und ihnen neue Handlungsoptionen verschaffen.

Aber nicht nur Mitarbeiter, auch Bürger müssen für Koproduktion und Co-Design geschult und bei Beteiligungsprozessen unterstützt werden. Die Herausforderung für Einrichtungen liegt auch darin, Bürgerinnen und Bürger als Unterstützer und Mitwirkende zu finden und sie an die Organisation $\mathrm{zu}$ binden. Ein professionelles Stakeholder-Management, das sich nicht nur um die Rekrutierung von Nutzern und Unterstützern kümmert, sondern auch um die Pflege derselben, ist dringend notwendig.
Um für Bürger attraktiv zu sein, die sich freiwillig engagieren möchten, ist eine breite Auswahl an Mitmachmöglichkeiten, die genau spezifiziert werden sollten, wichtig. Derzeit sind aber noch viele Organisationen im Dritten Sektor und auf staatlicher Seite nicht in der Lage, auf die unterschiedlichen Interessen der Bürger mit entsprechend diversifizierten Angeboten zu reagieren. In der Regel sind nicht einmal die Interessen der Bürger genau bekannt, so dass es für Organisationen schwierig wird, passgenaue Angebote zu machen (Bovaird/Löffler 2012, 58). Wenn Koproduktion und Co-Design sich in einer Kommune nicht ausbreiten, dann auch deshalb, weil es den Organisationen dort nicht gelingt, an die Interessen von Bürgerinnen und Bürgern anzudocken.

Zu den größten Hürden, die es für die Koproduktion zu nehmen gilt, zählen die Leistungsvereinbarungen, die soziale Dienste mit der öffentlichen Hand und den Kostenträgern schließen. In diesen Vereinbarungen werden - zumeist sehr detailliert - Art, Umfang und Vergütung sozialer Dienstleistungen festgelegt. An diesen Kontraktverhandlungen zwischen Staat und freien Trägern sind Bürger nicht beteiligt. In den Kontrakten selbst wird die Mitsprache von Bürgern an der Leistungsausgestaltung nicht erwähnt. Immerhin können Nutzer und ihre Angehörige in Qualitätsdialoge einbezogen werden (BRV Jug, Anlage B, Teil E, Pkt. 3). Dennoch werden durch detaillierte Vorgaben Ideen der Bürger und offene Co-Designprozesse verhindert, denen man im Rahmen einer Outcome-Orientierung in den Verträgen eher Rechnung tragen könnte.

Um Koproduktion und Co-Design $\mathrm{zu}$ institutionalisieren, müssen entsprechende Vorgaben in die Rahmenverträge und örtlichen Leistungsvereinbarungen einfließen. Auf lokaler Ebene hat in Großbritannien Camden Town einen solchen Versuch unternommen und Koproduktions-Vorgaben in ihre Leistungsausschreibung für einen sozialen Dienst verfügt (nef 2012, 5).

Auch Australien will im öffentlichen Sektor neue Wege gehen und das CoDesign etablieren, um Koproduktion für Bürger attraktiv zu machen. Co-Design soll speziell in jenen Bereichen wie den sozialen Diensten eine Rolle spielen, in denen die Beziehungen zwischen Bürgern und Staat komplexer sind und über standardisierbare Verfahren hinausgehen (Lenihan/Briggs 2011). Co-Design ist der australischen Regierung zufolge mehr als Konsultation und Kundenbefragung: »It means engaging with individuals and groups from the beginning to the end of the process. (Lenihan/ Briggs 2011, 35)

In Deutschland steht eine große Debatte über Koproduktion und Co-Design noch aus - aber sie wird unumgänglich werden. Zum einen fordern Bürger in vielen Bereichen mehr Mitsprache, zum andern forcieren knappe öffentliche Budgets und der demografische Wandel die Entwicklung neuer Wege bei der Leistungsgestaltung und Leistungserbringung im Sozialbereich.

\section{Literatur}

Bovaird, Tony/Löffler, Elke (2012): From Engagement to Co-Production, in: Pestoff, Victor/ Brandsen, Taco/Verschuere, Bram (Hg.): New Public Governance, the Third Sector and CoProduction, New York: Routledge, 35-6o. BRVJug (Berliner Rahmenvertrag für Hilfen in Einrichtungen und durch Dienste der Kinder- und Jugendhilfe, Anlage B, www.berlin.de/imperia/ $\mathrm{md} /$ content/sen-jugend/rechtsvorschriften/brvj/ brvjug_anlage_b.pdf?start\&ts=1279280388\&file =brvjug_anlage_b.pdf [abgerufen am 16.5.2013]. Hagen, Penny/Robertson, Toni (2012): Social technologies: the Changing Nature of Participation in Design, in: Designlssues, vol. 28, Heft 3, 77-88.

Lenihan, Don/Briggs, Lynelle (2011): Co-Design: Toward a New Service Vision for Australia?, in: Public Administration Today, Heft 25, 35-47, www.humanservices.gov.au/spw/corporate/ publications-and-resources/resources/co-design-toward-a-new-service-vision-for-australia. pdf [abgerufen am 16.5.2013].

New Economics Foundation (2012): Action Points for developing co-production in social care, in: Budgets and Beyond (Briefing 2), www.thinklocalactpersonal.org.uk/_library/Resources/Budgets_ and_Beyond_2.pdf [abgerufen am 16.5.2013]. Reiser, Brigitte (2012): Für eine Kultur der Mitgestaltung. Freiwilligendienste und bürgerschaftliches Engagement fordern gemeinnützige Organisationen heraus, in: Blätter der Wohlfahrtspflege, Jg. 159, Heft 6, 206-208.

Sanders, Elizabeth B.N./Stappers, Pieter Jan (2008): Co-creation and the new landscapes of design, in: CoDesign, vol. 4, Heft 1, 15-18. 